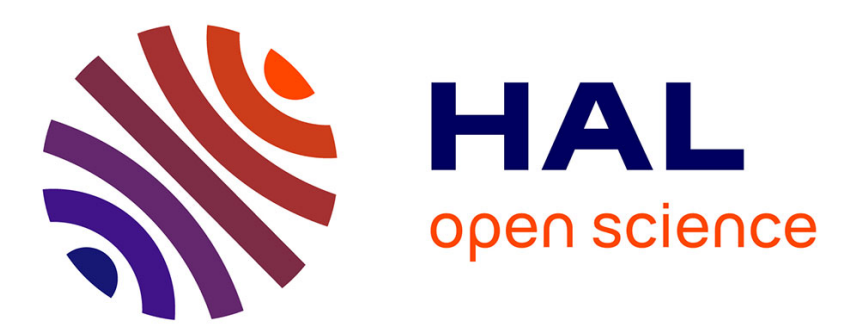

\title{
Damage fluctuations in creep deformed copper studied with synchrotron X-ray microtomography
}

Ramin Abbasi, Loïc Renversade, Kristof Dzieciol, András Borbély, Anke R. Kaysser-Pyzalla

\section{To cite this version:}

Ramin Abbasi, Loïc Renversade, Kristof Dzieciol, András Borbély, Anke R. Kaysser-Pyzalla. Damage fluctuations in creep deformed copper studied with synchrotron X-ray microtomography. International Journal of Materials Research, 2012, 103 (2), pp.228-233. 10.3139/146.110662 . hal-00818284

\section{HAL Id: hal-00818284 \\ https://hal.science/hal-00818284}

Submitted on 13 Sep 2021

HAL is a multi-disciplinary open access archive for the deposit and dissemination of scientific research documents, whether they are published or not. The documents may come from teaching and research institutions in France or abroad, or from public or private research centers.
L'archive ouverte pluridisciplinaire HAL, est destinée au dépôt et à la diffusion de documents scientifiques de niveau recherche, publiés ou non, émanant des établissements d'enseignement et de recherche français ou étrangers, des laboratoires publics ou privés. 


\title{
Damage fluctuationsin creep deformed copper studied withsynchrotron X-ray microtomography
}

\author{
Ramin Abbasia ${ }^{a}$ Loïc Renversade ${ }^{\mathrm{a}}$, Krzysztof Dzieciola ${ }^{\mathrm{a}}$, András Borbély ${ }^{\mathrm{a}}$, \\ Anke R. Kaysser-Pyzalla ${ }^{b}$ \\ ${ }^{a}$ Centre SMS, École Nationale Supérieure des Mines de Saint-Étienne, Saint-Étienne, France \\ ${ }^{\mathrm{b}}$ Helmholtz Zentrum für Materialien und Energie GmbH, Berlin, Germany
}

\begin{abstract}
Damage localization during power law creep of copper has been investigated in situ with synchrotron X-ray microtomography. The analysis of the area fraction of cavities corresponding to a given material slice has revealed that damage localization begins relatively early at the half of the creep lifetime. The amplitude of the maximum fluctuation shows parabolic behavior as a function of mean void volume fraction. Existing models of damage evolution underpredict the amount of real damage and overpredict powerlaw creep life-time.
\end{abstract}

Keywords: Creep damage; Damage fluctuations; Microtomography; Void growth

\section{Introduction}

Fracture of metals and alloys during creep at elevated temperatures occurs generally by nucleation, growth and coalescence of grain boundary voids. Since the nucleation process is taking place at the nanometer scale or below, present day characterization techniques allow a more adequate description of void growth and coalescence. Our present experimental knowledge on void growth is summarized in creep damage mechanism maps [1], similar to the wellknown deformation mechanism maps [2]. There are three basic mechanisms of void growth that depend on deformation conditions [3]: at low strain rates and stresses the diffusive mechanism dominates $[4,5]$, while at high rates and stresses growth becomes controlled by plastic flow [6, 7]. At intermediate values a coupling between diffusive growth and plastic flow is expected [8,9], but the mechanism has never been observed experimentally in spite of several attempts [10]. Present work focuses on the plastic creep mechanism characteristic for deformation conditions imposed in the experiment. For the same samples presented here, Dzieciol et al. [11] have shown that growth mechanism of cavities is viscous flow and was identified with the glide and climb mechanisms of dislocations. Considering the viscous material flow for creep Cocks and Ashby [7] have developed a general continuum model, which predicts the time evolution of the area fraction of the voids prevailing on sample cross-sections lying perpendicular to the externally applied stress. The model emphasizes that damage evolution is deterministic, in the sense that material sections with initially higher amount of damage have higher damage rates. The aim of the present work is to study the local evolution of the area fraction of voids and to compare it with predictions of the existing model [7].

Experimental investigation of damage evolution is a challenging task since, as mentioned above, the damage rate depends on the amount of damage present in the material. This makes difficult the comparison between results obtained on different specimens by destructive techniques such as optical or electron microscopy. Moreover, damage assessment at the surface of the material can be quite different from damage in the bulk, for which reason application of non-destructive investigation methods capable of capturing these changes is desirable. Among several methods able to monitor damage we mention acoustic emission (AE) which is a standard qualitative technique [12] as well as synchrotron X-ray microtomography that opened new perspectives for three-dimensional quantitative characterization of heterogeneous materials [13-17]. Microtomography has been already applied to in-situ characterization of creep damage $[11,15,18]$ and the results show that the growth rate of single cavities in copper [11] and brass [18] is much faster than predictions of the continuum theory [3, 19]. Compared to our previous work focusing on cavity growth mechanisms at the single void level [11], here we expand the scale of analysis to the level of sample size (in directions perpendicular to the externally applied stress). Along the sample length, however, we keep the micrometer resolution to better describe damage localization. The analysis of damage at the lengthscale of the sample's size allows a novel treatment of the experimental data from the point of view of a statistical system with monotonically increasing fluctuations.

\section{Experimental procedure}

Cylindrical specimens of $1 \mathrm{~mm}$ in diameter and $1 \mathrm{~mm}$ in gauge length were creep deformed under uniaxial tension using a miniaturized creep machine mounted on the goniometer used for tomography scanning (a detailed description of the setup can be found in Ref. [20]). In-situ monitoring of sample deformation was made with an inductive displacement transducer, however, the local strain and strain-rate of the samples was evaluated from the 3D tomo- 

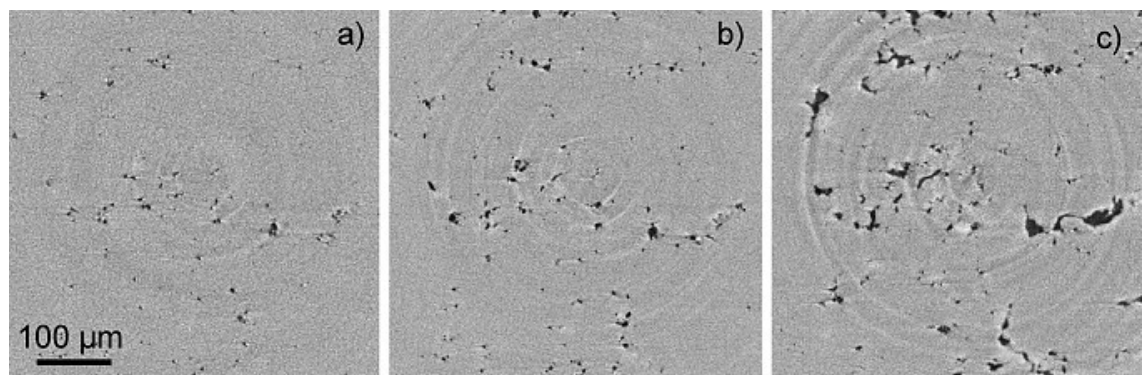

Fig. 1. Damage evolution on slice no. 56 (Fig. 2) having the highest void area fraction in the last reconstructed state of the sample deformed at $773 \mathrm{~K}$. (a) $t=0.36 t_{\mathrm{f}}$, (b) $t=0.53 t_{\mathrm{f}}$ and (c) $t=0.79 t_{\mathrm{f}}$. The creep time is indicated as part of the failure time $t_{\mathrm{f}}$.

graphic reconstructions through image correlation [21]. The force necessary for deformation was supplied by two springs and was measured with a load cell. The samples were heated and kept at high temperature by two resistive coils positioned at the top and at the bottom part of the gauge. Three samples made of oxygen free high conductivity copper (OFHC) were creep deformed at temperatures of $573 \mathrm{~K}, 723 \mathrm{~K}$ and $773 \mathrm{~K}$, respectively.

Microtomography was performed in situ at ID 15A of the ESRF using an intense and parallel synchrotron beam generated by an undulator source. The mean energy of the beam was of about $80 \mathrm{keV}$ and had a large band-width. The transmitted intensity was recorded using an X-ray detector with an effective pixel size of $1.4 \times 1.4 \mu \mathrm{m}^{2}$. The CCD of the detector with a resolution of about $2.1 \mu \mathrm{m}$ [22] was working in frame transfer mode. The readout speed of 60 frames per second of the CCD allowed operation without mechanical shutter. Motorized slits defined the horizontal and vertical beam sizes of $1.7 \mathrm{~mm}$ and $1.3 \mathrm{~mm}$, respectively. 800 radiographs with an exposure time of $250 \mathrm{~ms}$ each were collected in about $3 \mathrm{~min}$ and scans were performed every $15 \mathrm{~min}$.

\section{Results}

3D tomographic scans were reconstructed using in-house developed software based on the filtered back projection algorithm and accounting for the specific noise present in the measurement. The images were segmented with the watershed algorithm using markers originating from a local threshold criterion [23]. Damage was characterized by the area fraction of voids $A_{A}$, on slices lying perpendicular to the external stress. The evolution of $A_{A}$ on each material slice has been followed through image registration by cross correlating slices of consecutively reconstructed volumes [21]. Figure 1 shows, for example, void evolution in a region of interest containing the most damaged slice (in the last reconstructed state) corresponding to the sample deformed at $773 \mathrm{~K}$. The area fraction of cavities for all registered material slices is shown in Fig. 2 as a function of the slice number in the initial state and at different reduced creep times (creep time as multiple of creep lifetime). The figure indicates well damage localization by the gradually increasing local amplitudes. We should emphasize that remarkable fluctuations are already present at the beginning of secondary creep regime $\left(0.36 t_{\mathrm{f}}\right)$.

The results of slice registration can be used for the evaluation of the local strain and strain rate in the sample, too. Selecting an initial reference distance along the $Z$ axis of $l_{0}=50$ layers $(70 \mu \mathrm{m})$, the results of slice correlation will also provide the length $l$ between selected reference slices. The local true strain is simply obtained as: $\varepsilon_{\mathrm{loc}}=\ln \frac{l}{l_{0}}$

The strain rate is obtained in a second step by dividing the strain with the time interval between cross-correlated reconstructions, typically of $30 \mathrm{~min}$. Figure 3 shows the distribution of the local strain rate (left axis) along the gauge length of the sample deformed at $773 \mathrm{~K}$ at two characteristic points of the creep curve: a) at the middle of secondary state and b) at the beginning of tertiary state. The strain rate

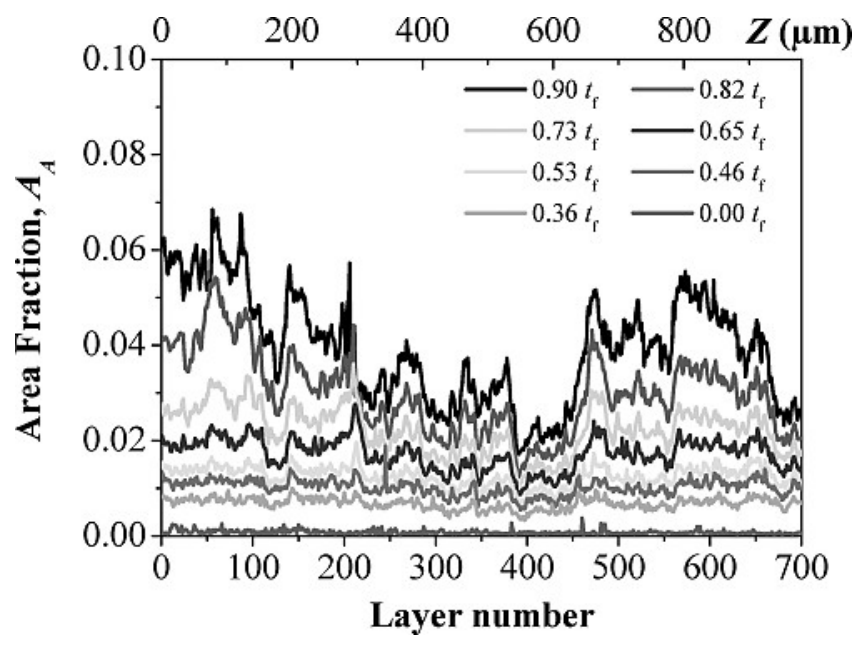

Fig. 2. Evolution of the area fraction of cavities on material slices lying perpendicular to the external load at different creep times. The slice number corresponds to the initial state. The upper horizontal scale gives the position of the slices in $\mu \mathrm{m}$ (sample deformed at $773 \mathrm{~K}$ ).

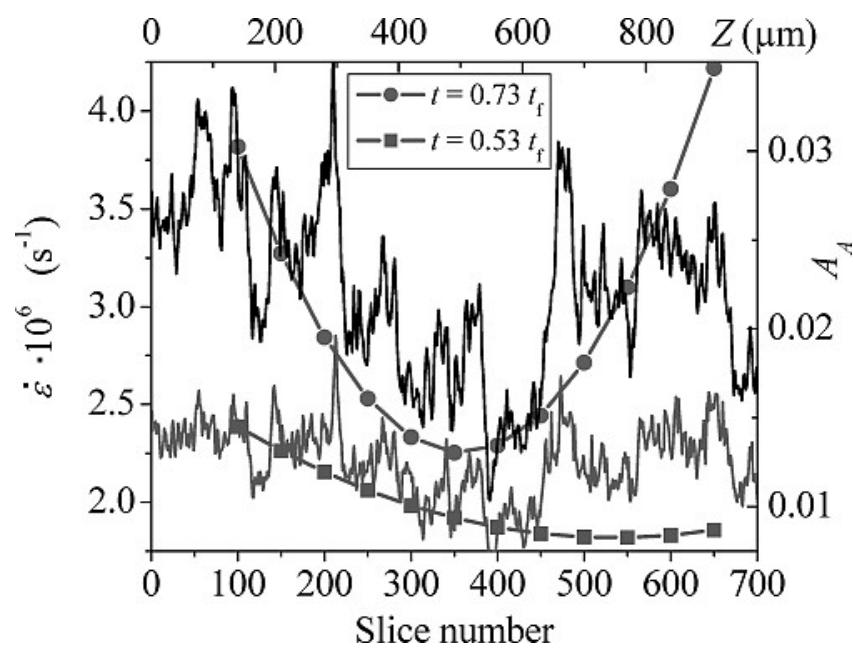

Fig. 3. Local strain rate along the gauge length at two deformation states $\left(t=0.53 t_{\mathrm{f}}\right.$ and $\left.t=0.73 t_{\mathrm{f}}\right)$ and the corresponding distribution of $A_{A}$. 
at $0.53 t_{\mathrm{f}}$ decreases slightly (by about $20 \%$ ) from layer 100 to layer 700. This decrease is larger than the error of the strain rate estimated from cross-correlation analysis to be of about $5 \%$. A possible reason for this could be the small temperature gradient pointing towards the bottom of the sample. At the beginning of the tertiary creep stage, however, the strain rate shows quite different behavior with higher values near the extremities of the gauge length. This is in good agreement with the variation of the average area fraction of voids (Fig. 3 right axis). The comparison emphasizes the strong coupling between local damage and local strain rate.

To describe damage localization the evolution of porosity on the material slice having the largest area fraction $A_{A \max }$, (in the last reconstructed state) was selected. In order to compare the samples creep deformed under different conditions, the average value of $\bar{A}_{A}$ (global porosity) was chosen as an independent variable. Figure 4 shows that the evolution of $A_{A \max }$ is very similar for the three samples not depending on temperature, strain rate or stress. This emphasizes that the damage mechanism (growth and coalescence of voids by viscous flow) is the same in each case. The largest area fraction of voids for the sample deformed at the lowest temperature $573 \mathrm{~K}$ and highest stress $87 \mathrm{MPa}$ is relatively small $(\sim 0.0075)$ since the last analyzed state is still in the secondary creep regime. The reason for this is the relatively long acquisition time of a tomographic scan ( $\sim 3 \mathrm{~min})$ compared to the actual strain rate. During the acquisition time the movement of material layers due to sample elongation is larger than the pixel size, which leads to blurring of the image. The last reconstructed state for this sample corresponds only to $75 \%$ of creep lifetime (see Fig. 6). For the case of samples deformed at $723 \mathrm{~K}$ and $773 \mathrm{~K}$ the last reconstructed state is in the tertiary stage at $88 \%$ and $90 \%$ of the lifetime, respectively.

It was suggested recently [12] that creep lifetime of composites is proportional to the duration of the primary creep regime. Such a discovery is very surprising and it can offer a good method of rupture prediction. Exploring the phenomena from a microstructural point of view, this discovery means that essential features of damage should already be present in the microstructure at the beginning of the secondary creep regime. It is evident that the copper polycrystal shows less heterogeneity than fiber reinforced composites [12], but as shown previously (Fig. 2) one cannot exclude

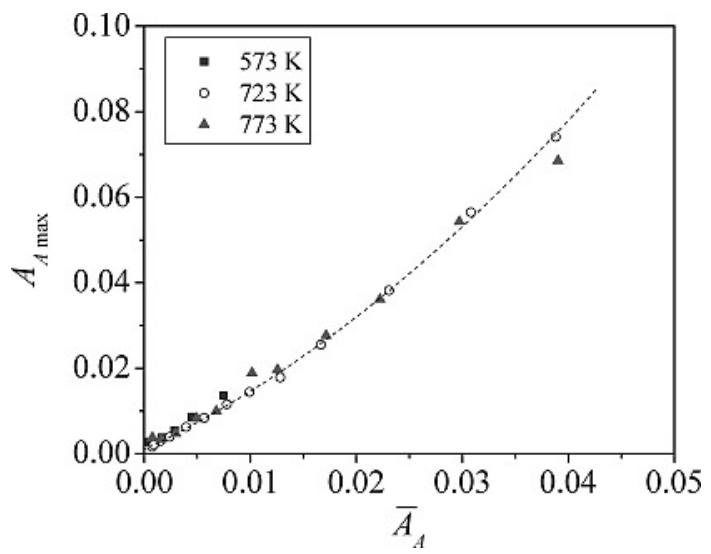

Fig. 4. Growth o fthe largest fluctuation $A_{A \max }$ as a function of the mean area fraction (volume fraction) of voids. that an initially present small heterogeneity of damage determines the final fracture and its location. Considering the distribution of $A_{A}$ in the final reconstructed state as representative for damage distribution before fracture we have quantified the similarity between this distribution and those at earlier deformation states. The corresponding cross correlation coefficients $C C$, are shown in Fig. 5a as a function of $\bar{A}_{A}$. All samples show monotonically increasing crosscorrelation coefficients indicating increasing similarity with the final state. It is remarkable the fast increase of the correlation coefficients corresponding to the sample deformed at the highest strain rate and lowest temperature ( $573 \mathrm{~K})$. The two other samples $(723 \mathrm{~K}$ and $773 \mathrm{~K})$ having nearly identical strain rate show very similar variations, which means that the strain rate is an important the parameter affecting localization. Choosing a threshold value for the correlation coefficient it becomes possible identifying a previous deformation state, in which damage distribution already showed a strong similarity to that before rupture. For the usual "strong correlation" threshold of $C C_{\text {th }}=0.7$, the corresponding "similar" states are indicated by circles (R) in Fig. $5 b$ (the states before rupture are indicated by F). It is remarkable that for all samples the state with high similarity already appears in the secondary stage (half of the lifetime), when the corresponding creep strain is only about $5 \%$. The creep curves in Fig. $5 \mathrm{~b}$ were calculated from the measured displacement of samples top using the inductive displacement transducer. Strain calculation was done choosing the effective length $l_{\text {eff }}=2.3 \mathrm{~mm}$, which led to
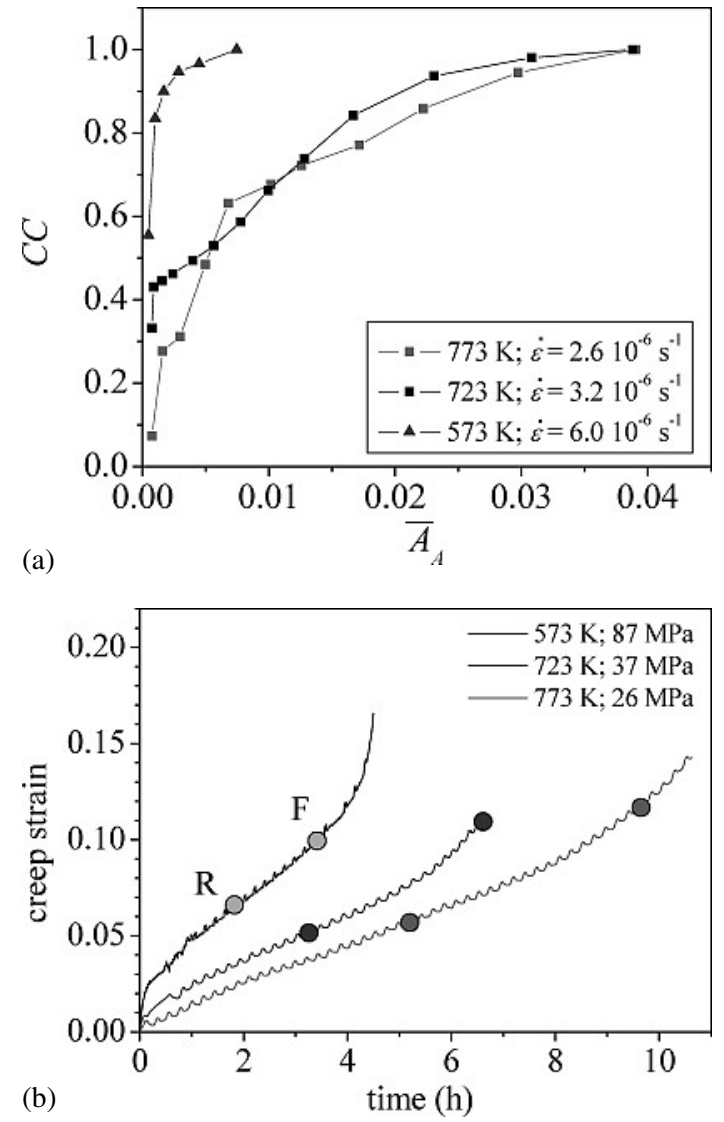

Fig. 5. (a) Variation of the cross-correlation coefficient between the final and previous distributions of $A_{A}$ as a function of the average area fraction of voids, and (b) corrected average creep curves using an effective sample length of $2.3 \mathrm{~mm}$. 
the same value of the average strain rate in the secondary stage as the value obtained from tomographic evaluations. The undulations on the curves originate from the inductive displacement transducer, the cables of which were moving during the tomographic scan.

In Ref. [11] a comparison between the evolution of experimentally determined mean volume fraction of cavities and the prediction of the Cocks and Ashby model [7] was presented. Here we use the Cocks and Ashby model to predict the evolution of damage fluctuations (Eq. (16) of [7]). Since the model has been developed for the case of non-interacting voids such a comparison allows quantifying the importance of cavity interaction, which is expected to become more and more important at later stages of creep. Therefore the evolution of $A_{A}$ on each material section was calculated based on the Cocks and Ashby model [7] starting from initial values corresponding to the "strongly similar" states identified by cross-correlation. The results show first of all that local damage evolution is faster in experiment than in simulation in agreement with previous findings for $\bar{A}_{A}$ [11]. To better emphasize the similarities between experimental and predicted distributions of $A_{A}$ the calculations were continued for a longer time until the model curves reached one point on the measured curves. As shown in the Fig. 6 the predicted damage distributions describe satisfactorily the measured experimental distribution. Exceptions are the regions with strong localization of $A_{A}$.

\section{Discussion}

There are a relatively small number of scientific publications dealing with in-situ analysis of damage localization during plastic deformation of engineering materials [11, $12,15,18,25]$. The existing tomographic data describe the different type of damage in complex composite structures during room temperature (RT) tensile deformation $[16,24]$ or creep at high temperature [25]. Tomographic reconstructions of these composites clearly evidence different damage mechanisms, such as particle delamination, particle fracture, void formation and growth at various stages of creep $[16,25]$. This earlier work has mainly focused on describing damage evolution at the micro-scale in terms of the average void [15, 26, 27] and in terms of single cavities $[11,18]$. Another parameter investigated was the mean volume fraction of voids or global porosity, which for RT deformation was found to increase much slower with deformation [24] than the prediction of the classical Rice and Tracey approach [28]. Good agreement has been found, however, between the predicted and experimentally determined local porosity for some sub-volumes of the structure [24]. This clearly indicates damage inhomogeneity and the inadequacy of a mean field approach. Dzieciol et al. [11] have described this inhomogeneous distribution of damage in terms of the area fraction of voids on planes perpendicular to the applied stress, which is probably the best choice if the cylindrical symmetry of the deformed sample is taken into account. The present work further expands the latter study by analyzing the evolution of damage fluctuations and trying to answer questions related to damage localization and final fracture.

The present results indicated that a strong coupling exists between the amount of local porosity and the local creep rate. It is interesting to note that variations of about $1 \%$ in the area fraction between different parts of the gauge length (Fig. 3) lead to relatively large variation of the local strain rate. This self-amplifying process is characteristic for tertiary creep stage. The origin of damage localization roots, however, much deeper in the void growth mechanism. For viscous flow the volumetric void growth rate is proportional to void volume [19], which means that larger voids grow faster than smaller ones. Thanks to the sensitivity of the tomographic evaluation the growth of small fluctuations is already observable in the secondary stage of creep.
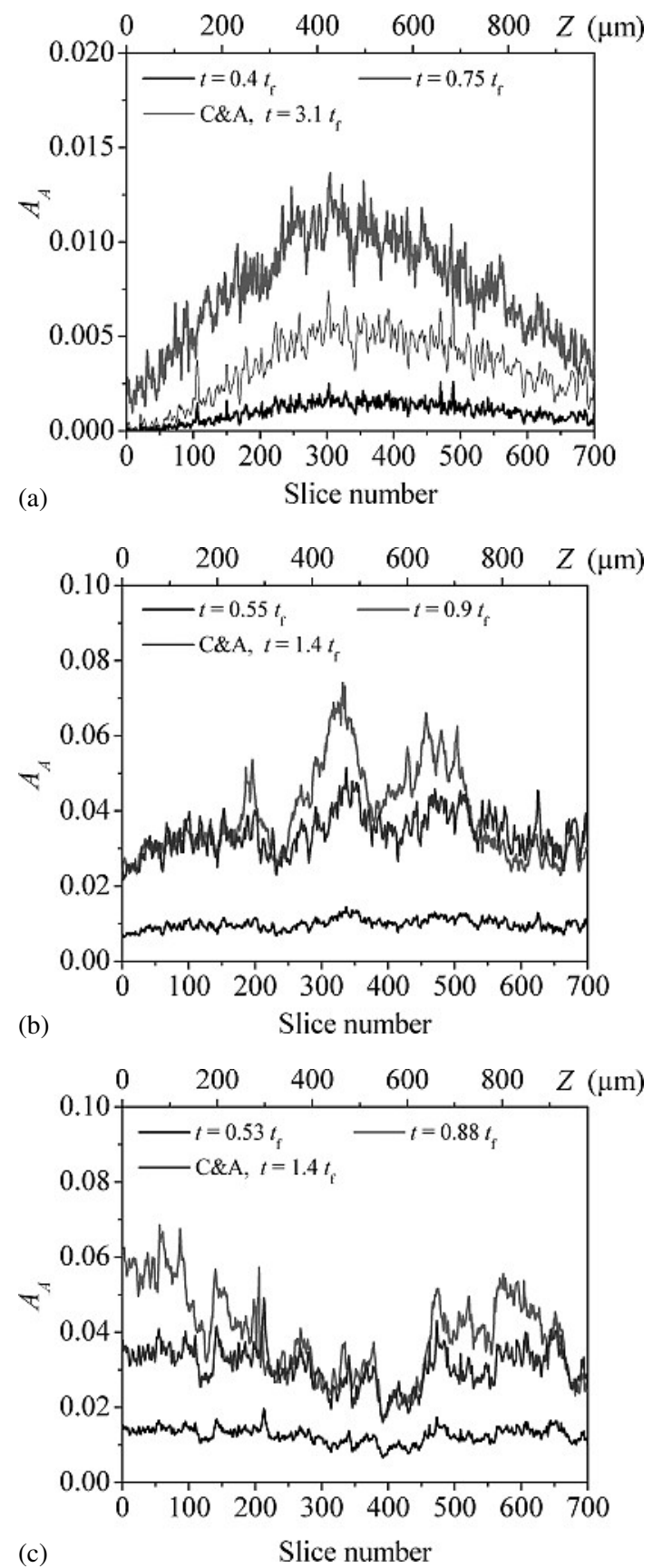

Fig. 6. Comparison between experimentally determined and predicted distributions of void area fraction along the gauge length. The experimental distributions correspond to the final reconstructed state $(\mathrm{F})$ and a previous reference state $(\mathrm{R})$ with a cross-correlation coefficient larger than 0.7 . The reference state has been used as input for prediction of damage distribution according to the model of Cocks and Ashby [7]. (a) sample deformed at $573 \mathrm{~K}$, (b) at $723 \mathrm{~K}$ and (c) at $773 \mathrm{~K}$. 
From the point of view of creep lifetime prediction the most interesting parameter seems to be the fluctuation having the maximum amplitude $A_{A \max }$. How this amplitude is related to other structural parameters is a very intriguing question. Obtaining such a relationship could serve as a good predictor of creep lifetime once an analysis of the microstructure has been performed. We have found empirically a parabolic law describing the evolution of $A_{A \max }$ as a function of the mean volume fraction $\bar{A}_{A}$ :

$A_{A \max }=a_{0}+a_{1}\left(\bar{A}_{A}\right)+a_{2}\left(\bar{A}_{A}\right)^{2}$

where $\quad a_{0}=10^{-3} \pm 3 \cdot 10^{-4}, \quad a_{1}=1.18 \pm 0.06 \quad$ and $a_{2}=18.6 \pm 1.5$. As fluctuations depend on the size of the system Eq. (2) should be considered valid only for copper specimens with dimensions similar to the ones investigated in present work. We are also aware that the global porosity is not the best independent parameter, but the independence of $A_{A \max }$ from temperature and strain rate indicates that this approach might be adapted to practical cases with well-defined geometry and where creep lifetime prediction is an important issue. It is important to note the relatively small value of $A_{A \max } \cong 8 \%$ in the last reconstructed state $\left(t \cong 0.9 t_{\mathrm{f}}\right)$. This is much smaller than the value of $25 \%$ usually considered as a failure criterion [7].

Cross-correlation results between the distribution of in the last reconstructed state and earlier states revealed a relatively new aspect of damage localization. The strong correlation $(C C>0.7)$ between the distributions at about $90 \%$ and $50 \%$ of creep lifetime is remarkable, which clearly indicates again the deterministic nature of damage evolution. Compared to results originating from the macroscopic behavior of the creep curves and relating fracture time to the length of the primary creep regime [12], the present data show that damage distribution before rupture is strongly correlated to a state near the middle of the secondary stage. As indicated by the rate of AE energy release substantial damage in composites has already been formed at the end of primary creep, which might explain the discovered correlation with the creep lifetime [12]. Contrary to this, in the copper polycrystal damage evolves more progressively, dominant fluctuations developing only in the secondary stage. The corresponding creep time is about half of the creep lifetime, but is also surprisingly small. In line with previous findings simulations of damage distribution according to the model of Cocks and Ashby [7] have been performed. The calculations show that theoretical predictions and experimental data are in satisfactory agreement (Fig. 6) suggesting that damage distribution at the half of the creep lifetime is already characteristic for that in the final state. The model does not adequately describe damage growth in regions of high localization. This faster growth in experiments can be attributed to void interaction effects not taken into account by the model. Evidently, the results for the sample deformed at the lowest temperature having the last reconstructed state at $75 \%$ of the lifetime are less characteristic than those of the other two with final reconstructions at $0.88 t_{\mathrm{f}}$ and $0.90 t_{\mathrm{f}}$, respectively. Ideally, damage distribution in a state as near as possible to the moment of the final fracture is needed. This requires faster tomographic scans, which will probably be available with a new generation of CMOS based X-ray detectors.

\section{Conclusions}

Creep damage localization in terms of the area fraction of voids on slices perpendicular to the external stress was investigated with in-situ tomography. It was found that:

- damage localization begins relatively early; distribution of $A_{\mathrm{A}}$ along the gauge length of the sample at half of the creep lifetime shows already strong correlation with the distribution at $t \cong 0.9 t_{\mathrm{f}}$.

- the amplitude of the maximum fluctuation increases parabolically as a function of the average area fraction of voids. For power-law creep of copper this relationship is independent of temperature, stress or strain rate.

The authors acknowledge the help of the ID-15 beamline staff (ESRF).

\section{References}

[1] A.C.F. Cocks, M.F. Ashby: Prog. Mater. Sci. 27 (1982) 189. DOI:10.1016/0079-6425(82)90001-9

[2] H.J. Frost, M.F. Ashby: Deformation-Mechanism Maps, The Plasticity and Creep of Metals and Ceramics, Pergamon Press, Oxford Oxfordshire and New York (1982).

[3] H. Riedel: Fracture at High Temperatures, Springer-Verlag, Berlin (1987).

[4] D. Hull, D.E. Rimmer: Phil. Mag. 4 (1959) 673. DOI:10.1080/14786435908243264

[5] M.V. Speight, W. Beere: Metal Sci. 9 (1975) 190. DOI: $10.1179 / 030634575790445161$

[6] J.W. Hankock: Metal Sci. 10 (1976) 319.

[7] A.C.F. Cocks, M.F. Ashby: Metal Sci. 14 (1980) 395. DOI:10.1016/0036-9748(80)90333-6

[8] A. Needleman, R. Rice: Acta Metall. 28 (1980) 1315. DOI:10.1016/0001-6160(80)90001-2

[9] I.W. Chen, A.S. Argon: Acta Metall. 29 (1981) 1759. DOI: $10.1016 / 0001-6160(81) 90009-2$

[10] J.S. Wang, L. Martinez, W.D. Nix: Acta Metall. 31 (1983) 783. DOI:10.1016/0001-6160(83)90115-3

[11] K. Dzieciol, A. Borbély, F. Sket, A. Isaac, M. Di Michiel, P. Cloetens, Th. Buslaps, A.R. Kaysser-Pyzalla: Acta Mater. 59 (2011) 671. DOI:10.1016/j.actamat.2010.10.003

[12] H. Nechad, A. Helmstetter, R. El Guerjouma, D. Sornette: Phys. Rev. Lett. 94 (2005) 045501. DOI:10.1103/PhysRevLett.94.045501

[13] B.P. Flannery, H.W. Deckman, W.G. Roberge, K.L. D'Amico: Science 237 (1987) 1439. DOI:10.1126/science.237.4821.1439

[14] A. Borbély, F.F. Csikor, S. Zabler, P. Cloetens, H. Biermann: Mater. Sci. Eng. A 367 (2004) 4050.

[15] A.R. Pyzalla, B. Camin, Th. Buslaps, M. Di Michiel, H. Kaminski, A. Kottar, A. Pernack, W. Reimers: Science 305 (2005) 92. DOI: $10.1126 /$ science. 1106778

[16] E. Maire, V. Carmona, J. Courbon, W. Ludwig: Acta Materialia 55 (2007) 6806. DOI:10.1016/j.actamat.2007.08.043

[17] J. Banhart, A. Borbély, K. Dzieciol, F. Garcia-Moreno, I. Manke, N. Kardjilov, A.R. Kaysser-Pyzalla, M. Strobl, W. Treimer: Int. J. Mat. Res. 101 (2010) 9.

[18] A. Isaac, F. Sket, K. Dzieciol, A. Borbély: Met. Mat. Trans. A 42 (2011) 3022. DOI:10.1007/s11661-011-0781-1

[19] B. Budiansky, J.W. Hutchinson, S. Slutsky in: H.G. Hopkins, M.J. Sewell (Eds.) Mechanics of solids. The R. Hill $60^{\text {th }}$ anniversary volume, Pergamon, Oxford, (1982) p. 13.

[20] F. Sket, K. Dzieciol, A. Isaac, A. Borbély, A.R. Pyzalla: Mater. Sci. Eng. A 527 (2010) 2112. DOI:10.1016/j.msea.2009.11.058

[21] K. Dzieciol, A. Isaac, F. Sket, A. Borbély, A.R. Pyzalla: Proc. EPD Congress, San-Francisco, (2009), p. 15

[22] M. Di Michiel, J.M. Merino, D. Fernandez-Carreiras, Th. Buslaps, V. Honkimaki: Rev. Sci. Instrum. 76 (2005) 043702. DOI:10.1063/1.1884194

[23] M. Sezgin, B. Sankur: J. Electron. Imaging 13 (2004) 146. DOI: $10.1117 / 1.1631315$

[24] L. Babout, E. Maire, J.Y. Buffière, R. Fougères: Acta Mater. 49 (2001) 2055. DOI:10.1016/S1359-6454(01)00104-5

[25] A. Pyzalla, B. Camin, B. Lehrer, M. Wichert, A. Koch, K. Zimnik, E. Boller, W. Reimers: JCPDS-International Centre for Diffraction Data (2006) ISSN 1097-0002, 73. 
[26] A. Isaac, F. Sket, W. Reimers, B. Camin, G. Sauthoff, A.R. Pyzalla: Mater. Sci. Eng. A 478 (2008) 108. DOI:10.1016/j.msea.2007.05.108

[27] E. Maire, O. Bouaziz, M. Di Michiel, C. Verdu: Acta Mater. 56 (2008) 4954. DOI:10.1016/j.actamat.2008.06.015

[28] J.R. Rice, D.M. Tracey: J. Mech. Phys. Solids 17 (1969) 201. DOI:10.1016/0022-5096(69)90033-7

\section{Correspondence address}

András Borbély,

158 cours Fauriel, 4200 Saint-Etienne, France

Tel.: +33477420279

Fax: +33477420001

E-mail: borbely@emse.fr 